\title{
Reforma integral para mejorar el desempeño del sistema de salud en México
}

\author{
Julio Frenk, PhD, (1) Eduardo González-Pier, PhD, (2) Octavio Gómez-Dantés, MPH, ${ }^{(2)}$ \\ Miguel Ángel Lezana, MD, ${ }^{(2)}$ Felicia Marie Knaul, PhD. ${ }^{(3)}$
}

\author{
Frenk J, González-Pier E, Gómez-Dantés O, \\ Lezana MA, Knaul FM. \\ Reforma integral para mejorar el desempeño \\ del sistema de salud en México. \\ Salud Publica Mex 2007;49 supl I:S23-S36.
}

\section{Resumen}

A pesar de haber alcanzado una esperanza de vida promedio de 75 años, similar a la de países más desarrollados, México ingresó al siglo XXI con un sistema de salud marcado por su incapacidad para ofrecer protección financiera en salud a más de la mitad de su población. Esto es resultado y causa de las desigualdades sociales que han caracterizado el proceso de desarrollo en México.Varias limitaciones estructurales han dificultado el funcionamiento y limitado el avance de su sistema de salud. Consciente de que la falta de protección financiera era su principal debilidad, México ha emprendido una reforma estructural para mejorar el desempeño del sistema de salud mediante el establecimiento del Sistema de Protección Social en Salud (SPSS), el cual ha introducido nuevas reglas de financiamiento e incentivos. La principal innovación de la reforma ha sido el Seguro Popular de Salud, el componente de aseguramiento del SPSS dirigido al financiamiento de la atención médica para todas aquellas familias, en su mayoría pobres, que históricamente habían sido excluidas de la seguridad social. La reforma ha permitido un incremento significativo en la inversión pública en salud, al tiempo que realinea los incentivos para garantizar una atención de mayor calidad técnica e interpersonal. En este trabajo se describen las principales características y los resultados iniciales de este esfuerzo de reforma de México, y se derivan algunas lecciones para otros países que consideren llevar a cabo transformaciones a su sistema de salud en circunstancias de desafío similares.

Palabras clave: protección financiera en salud; calidad de la atención en salud; México
Frenk J, González-Pier E, Gómez-Dantés O, Lezana MA, Knaul FM.

Comprehensive reform to improve health

system performance in Mexico.

Salud Publica Mex 2007;49 suppl I:S23-S36.

\section{Abstract}

Despite having achieved an average life expectancy of 75 years, much the same as that of more developed countries, Mexico entered the 2 Ist century with a health system mared by its failure to offer financial protection in health to more than half of its citizens; this was both a result and a cause of the social inequalities that have marked the development process in Mexico. Several structural limitations have hampered performance and limited the progress of the health system. Conscious that the lack of financial protection was the major bottleneck, Mexico has embarked on a structural reform to improve health system performance by establishing the System of Social Protection in Health (SSPH), which has introduced new financial rules and incentives. The main innovation of the reform has been the Seguro Popular (Popular Health Insurance), the insurance-based component of the SSPH, aimed at funding health care for all those families, most of them poor, who had been previously excluded from social health insurance. The reform has allowed for a substantial increase in public investment in health while realigning incentives towards better technical and interpersonal quality. This paper describes the main features and initial results of the Mexican reform effort, and derives lessons for other countries considering health-system transformations under similarly challenging circumstances.

Key words: financial protection in health; quality of health care; Mexico

(I) Fundación Bill y Melinda Gates.

(2) Secretaría de Salud, México.

(3) Fundación Mexicana para la Salud.

Solicitud de sobretiros: Felicia Marie Knaul, Fundación Mexicana para la Salud. Periférico Sur No. 4809, Col. El Arenal. I4610 Delegación Tlalpan México, DF, México.

Correo electrónico: knaul@prodigy.net.mx y fknaul@funsalud.org.mx 
E n todo el mundo, los países están innovando sus sistemas de salud como parte de la búsqueda continua de acceso universal, equidad, calidad y justicia en el financiamiento. En los últimos seis años, México ha avanzado en esta misma dirección. La actual reforma del sistema de salud está dirigida a los 50 millones de mexicanos no asegurados que habían sido excluidos de la seguridad social durante más de 60 años. La reforma estableció el Sistema de Protección Social en Salud (SPSS), el cual introdujo nuevas reglas para el financiamiento tanto de los servicios de salud pública y los servicios de salud dirigidos a la comunidad, como de los servicios personales de salud. Estos últimos se financian a través del Seguro Popular de Salud, el componente de aseguramiento subsidiado del SPSS que ofrece acceso gratuito en el momento de utilización de los servicios a un conjunto explícito de intervenciones de atención médica. En gran medida, la principal fuente de financiamiento proviene de impuestos federales y de contribuciones complementarias estatales. Las familias también realizan el pago anticipado de una pequeña prima definida de manera escalonada en función de su nivel de ingreso, de manera que el subsidio público es inversamente proporcional al ingreso familiar. El 20\% más pobre de las familias está exento de esta contribución. La población susceptible de afiliarse incluye a todas las personas, en su mayoría pobres, que no son beneficiarias de la seguridad social, ya sea porque son autoempleados, desempleados o están fuera de la fuerza de trabajo. La mayor parte son pobres y muchas viven en familias encabezadas por mujeres. Esto significa que la pobreza y la situación laboral de las personas ya no son obstáculo para acceder a alguno de los seguros de salud que ofrecen las instituciones públicas. La ley que establece el nuevo sistema fue aprobada en abril de 2003 y entró en vigor el $1^{\circ}$ de enero de 2004, con la meta de alcanzar la cobertura universal en materia de aseguramiento en salud en el año 2010.

Este trabajo, el primero de la serie sobre la reforma del sistema de salud mexicano, constituye una introducción a las principales características de esta reforma, y en esa medida proporciona antecedentes y evidencias para contextualizar los estudios analíticos y empíricos que le siguen. En Frenk ${ }^{1}$ se presentó ya un panorama general de la lógica y resultados de la reforma mexicana. En el presente trabajo describimos esta experiencia con mayor profundidad. Primero se presenta una breve descripción del sistema mexicano de salud, que incluye el origen y la organización de sus principales instituciones. Después se discuten los retos que enfrentaba el sistema mexicano de salud antes de la reforma. En seguida se presentan los fundamentos éticos y la visión de la reforma. A continuación se describe la manera en que se han modificado tres de las funciones clave del sistema de salud: rectoría, financiamiento y prestación de servicios. Más adelante se resumen los avances de la reforma en sus tres primeros años de implantación y se identifican algunos de los retos futuros. El trabajo finaliza con una síntesis de las lecciones aprendidas del proceso de reforma, las cuales pueden ser útiles para otros países que buscan mejorar el desempeño de sus sistemas de salud.

\section{Antecedentes del sistema mexicano de salud}

La fundación del sistema nacional de salud moderno data de 1943, año de creación de la Secretaría de Salud (SS), entonces Secretaría de Salubridad y Asistencia, y del Instituto Mexicano del Seguro Social (IMSS). En 1959, se crea el Instituto de Seguridad y Servicios Sociales para los Trabajadores del Estado (ISSSTE) para cubrir a los empleados del sector público y a sus familias. ${ }^{2}$

Durante este periodo, y hasta 2003, la población no cubierta por la seguridad social, principalmente la más pobre, era considerada como un grupo residual atendido por la SS con una fuente de financiamiento incierta y un paquete de beneficios mal definido. Esta población tenía acceso a unidades de servicios de salud operadas a nivel estatal y federal a cambio de un copago o cuota de recuperación en el momento de utilizar los servicios, que, a pesar de estar ajustada por el nivel de ingreso y contar con un subsidio significativo, contribuía a la alta proporción de gasto de bolsillo como fuente de financiamiento de la atención médica. La escasez de medicamentos en estas unidades médicas era común como resultado de limitaciones presupuestales y frecuentemente exponía a esta población a pagos de bolsillo adicionales. En contraste, el aseguramiento en salud en las instituciones de seguridad social garantiza el acceso gratuito a la atención a la salud en el momento de utilización de los servicios, y se financia con contribuciones de la nómina del empleador y del empleado, con una contribución adicional del gobierno federal que se financia a través de impuestos generales. En paralelo, existe un grupo heterogéneo y mal regulado de prestadores privados de servicios, muchos de ellos no certificados, que atendía y sigue atendiendo a las familias no aseguradas con capacidad de pago, y a la población que contaba con alguna seguridad social pero que estaba insatisfecha con la calidad de los servicios que ofrecían sus instituciones.

Es así que desde sus orígenes el sistema mexicano de salud ha estado organizado con base en un modelo segmentado, predominante en América Latina, marcado por la separación del derecho a la atención a la salud entre los asegurados del sector asalariado y for- 
mal de la economía y los no asegurados. Cada segmento de la población recibía los servicios de salud a través de instituciones integradas verticalmente, cada una de las cuales era responsable de la rectoría, el financiamiento y la prestación de servicios sólo para ese grupo de población (figura 1). ${ }^{2-5}$

Hasta el año 2000, antes de la reforma, el IMSS atendía a todos los trabajadores asalariados del sector privado y a sus familias, que representaban aproximadamente $40 \%$ de los casi 100 millones de habitantes de México; el ISSSTE atendía a 7\%, y los seguros privados cubrían de 3 a 4\% de la población. En consecuencia, aproximadamente $50 \%$ de la población no tenía acceso a ningún tipo de seguro médico prepagado. Esto incluía a alrededor de 2.5 millones de familias de los segmentos más pobres que recibían únicamente intervenciones de salud comunitarias y preventivas muy básicas incluidas en el programa de combate a la pobreza denominado Oportunidades. ${ }^{1}$

En general, prácticamente toda la población tenía acceso a algún tipo de servicio de salud, pero el número, calidad, efectividad y grado de protección financiera de las intervenciones variaban sustancialmente entre grupos de población, y eran particularmente deficientes para los pobres. ${ }^{6}$

\section{Retos enfrentados por la reforma}

$\mathrm{Al}$ igual que la mayoría de los países de ingresos medios, México experimenta procesos interrelacionados de transición demográfica y epidemiológica. La tasa de fecundidad declinó significativamente de 6.8 nacidos vi- vos por mujer en 1960 a 2.1 en 2005. Durante el mismo periodo, la esperanza de vida aumentó de 57.5 a 75.4 años. En consecuencia, la estructura de la población ha cambiado dramáticamente. Mientras que el grupo de menores de cinco años ha disminuido en números absolutos desde 1994, se espera que el crecimiento del grupo de la población de 60 años y más aumente a un ritmo de $4 \%$ anual en la próxima década. ${ }^{7,8}$

Aun cuando la transición epidemiológica está bastante avanzada, el proceso ha sido tanto complejo como prolongado: aunque las infecciones comunes han disminuido rápidamente, no han sido del todo controladas, mientras que las enfermedades no transmisibles y las lesiones ya constituyen las principales causas de mortalidad y discapacidad. ${ }^{1,9,10}$ Entre 1950 y 2000, la proporción de muertes atribuibles a enfermedades no transmisibles y lesiones aumentó de 44 a $73 \%$ y se espera que llegue a $78 \%$ en 2025 . En contraste, la proporción atribuible a enfermedades transmisibles disminuyó abruptamente de $50 \%$ en 1950 a $14 \%$ en 2000, y se espera que siga disminuyendo hasta representar $10 \%$ en $2025 .{ }^{11}$ En consecuencia, el sistema de salud se ha visto abrumado por la necesidad de enfrentar simultáneamente el rezago de las enfermedades transmisibles y los problemas de salud reproductiva que se concentran en la población más pobre y geográficamente dispersa, y por otro lado, el rápido incremento en la prevalencia de padecimientos no transmisibles y costosos que afectan a toda la población.

En 2000-2001, como parte del trabajo derivado del Programa Nacional de Salud actual, se identificaron

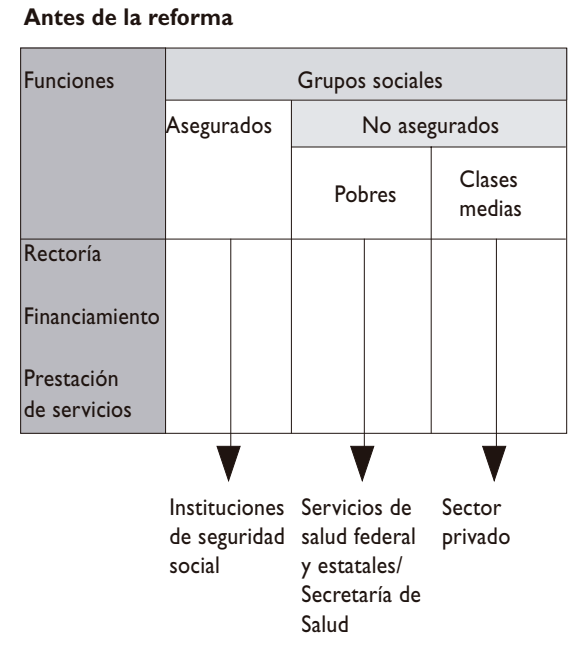

Fuente: Frenk J y colaboradores ${ }^{3}$

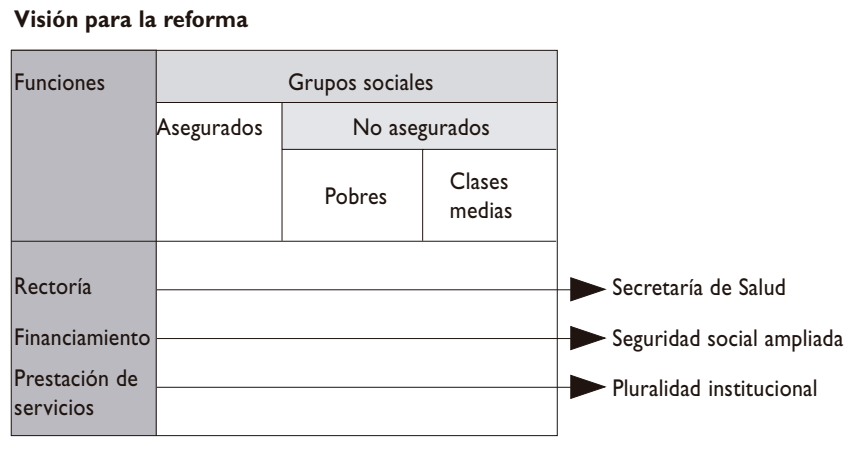

Figura I. Reorganización del sistema de salud mexicano 
cinco desequilibrios financieros como las principales limitantes que impedían que el sistema de salud respondiera a las necesidades de la población derivadas del desafío epidemiológico. ${ }^{11}$ Estos desequilibrios se relacionan con: i) un bajo nivel del gasto general en salud; ii) el predominio del gasto de bolsillo; iii) una asignación desigual de los recursos públicos entre los asegurados y los no asegurados, y entre estados; iv) una contribución estatal desigual al financiamiento de los servicios de salud, y v) una insuficiencia crónica de inversión en infraestructura de salud., 2,11,12

En primer lugar, el gasto total era insuficiente para un país con el nivel de desarrollo y la complejidad de necesidades de salud de México. En 2000, el país gastaba en servicios de salud sólo $5.6 \%$ del Producto Interno Bruto (PIB), alrededor de $\$ 350$ dólares per cápita, cifra muy por debajo del nivel promedio de América Latina de casi 7\%. En segundo lugar, a pesar de ser un medio ineficiente y desigual de financiar la salud, el gasto del bolsillo representaba la mayor parte del financiamiento total de la salud. Como se documenta en Knaul y colaboradores, en esta serie, dicha proporción es mayor que en muchos otros países con un nivel similar de desarrollo económico, y sustancialmente mayor que en países más desarrollados. ${ }^{13-15}$ El tercer desequilibrio se refiere a la distribución inequitativa de los fondos públicos entre grupos poblacionales y entre estados. Aun cuando los no asegurados representaban $55 \%$ de la población, en 2002 recibieron $34 \%$ del financiamiento público federal para salud. Esto se traduce en un nivel promedio de gasto público per cápita 2.3 veces mayor para los asegurados que para los no asegurados. Asimismo, el gasto federal per cápita varía entre las 32 entidades en el orden de 5 a 1 entre el estado con mayor gasto y el estado con la menor asignación. Las diferencias entre las aportaciones estatales per cápita en el mismo año fueron todavía más dramáticas, en el orden de 115 a 1. Finalmente, el gasto en equipamiento y nuevas instalaciones representó cuando mucho $2 \%$ del gasto federal total para los no asegurados. ${ }^{11-15}$

La dependencia del gasto de bolsillo colocó a las familias ante el grave riesgo de empobrecimiento, como se discute en Knaul y colaboradores y Gakidou y colaboradores en esta serie. ${ }^{13,16}$ De hecho, los análisis realizados por la Fundación Mexicana para la Salud (Funsalud) y la Secretaría de Salud han mostrado que al año, entre dos y cuatro millones de hogares mexicanos -la mayoría pobres y no asegurados- incurrían en gastos catastróficos (definidos como un porcentaje, por lo general $30 \%$, del ingreso total de la familia una vez descontado el gasto en alimentos) y gastos empobrecedores por motivos de salud (se refiere al gasto que empuja el ingreso del hogar por de- bajo o más abajo de un umbral, por lo general medido en términos de una línea de pobreza). ${ }^{17}$

El origen de las evidencias sobre estos desequilibrios se remonta a mediados de la década de los noventa y demuestra la importante contribución realizada por las instituciones de investigación locales. ${ }^{13,18}$ Esta capacidad local es el resultado de inversiones clave realizadas a lo largo de dos décadas para consolidar y ampliar la base del conocimiento en políticas de salud. Mediante el uso de diversas herramientas conceptuales y metodológicas desarrolladas por organizaciones académicas e internacionales, y gracias al trabajo con otras dependencias nacionales como el Instituto Nacional de Salud Pública, Funsalud construyó un sistema de cuentas nacionales de salud. El análisis realizado, dirigido hacia las políticas públicas, reveló la dependencia del gasto de bolsillo para financiar el sistema de salud. Estos hallazgos se reforzaron con la evaluación comparativa de los sistemas nacionales de salud, publicada como parte del Informe Mundial de la Salud de 2000, que identificó desigualdades sustanciales en el financiamiento de la salud en México. ${ }^{19}$ Este resultado motivó análisis adicionales detallados en el ámbito nacional por parte de Funsalud y la Secretaría de Salud. ${ }^{15,17}$

En suma, el grueso de las evidencias derivadas de estos análisis nacionales e internacionales, junto con la creciente presión de las cambiantes necesidades de salud de la población y las limitaciones del actual sistema de salud, confirmaron la necesidad de emprender una reforma de fondo. La esencia de esta reforma es la creación del SPSS y su componente de aseguramiento médico, el Seguro Popular de Salud (en adelante Seguro Popular).

A lo largo del proceso de reforma, los argumentos para su defensa, generados por el trabajo analítico sobre el financiamiento de la salud, jugaron un papel clave. El uso de la evidencia se reforzó con la aplicación de un marco ético explícito. Por ejemplo, mostrar que la población asegurada recibía muchos más recursos públicos que los pobres no asegurados permitió argumentar que el sistema actual violaba el principio democrático relativo a la igualdad de derechos de todos los ciudadanos.

\section{Fundamento ético}

Incluso cuando la Constitución mexicana reconoció formalmente el derecho social a la protección de la salud hace dos décadas, en la práctica, no todas las personas habían tenido la misma capacidad para ejercerlo. ${ }^{12}$ Como se indicó anteriormente, la mitad de la población, en virtud de su situación laboral, gozaba de la protección de la seguridad social y por lo tanto enfren- 
taba menos obstáculos al acceso a los servicios médicos que la mitad no asegurada.

Este tipo de segregación ocupacional es incompatible con la idea de que el acceso a la atención médica es un derecho humano, según lo estipula el Pacto Internacional de Derechos Económicos, Sociales y Culturales de Naciones Unidas. Mientras que México logró avanzar en el ejercicio de los derechos políticos y civiles como resultado de su proceso de democratización, resultaba claro que el siguiente gran reto era mejorar la desigualdad social mediante la garantía del ejercicio universal del derecho a los servicios de salud. En este contexto el término "universal" tiene dos significados: cubrir a todos y hacerlo sin ningún tipo de discriminación. Esto es especialmente importante para aquellos grupos que han sido excluidos de participar regularmente en el sector formal (por ejemplo trabajadores migrantes y hogares encabezados por mujeres). Éste fue el marco ético primordial en el cual se presentó la reforma.

La visión detrás de la reforma a la Ley General de Salud de 2003 es reorganizar el sistema de salud a través de la integración horizontal de sus tres funciones básicas: rectoría, financiamiento y prestación de servicios. Esta visión rompe con la situación previa a la reforma que fragmentaba el sistema verticalmente por grupos poblacionales (figura 1). Al contrario, la reforma se diseñó para reforzar el papel rector de la Secretaría de Salud.

Hasta la fecha, la reforma ha dado pasos importantes en esta dirección. En términos de rectoría, la reforma fortalece la función de la SS, la cual incluye el monitoreo, la evaluación del desempeño y la regulación de todo el sistema de salud. Asimismo, homogeneiza y hace universal el derecho de los ciudadanos al financiamiento público para la protección de la salud, y empodera a los consumidores para influir sobre la prestación de servicios de salud, a la vez que fortalece la oferta del sistema de salud. En seguida se describe la manera en que la reforma mexicana ha transformado estas tres funciones críticas de todo sistema de salud. ${ }^{20,21}$

\section{Principales componentes de la reforma}

\section{Rectoría}

La devolución y descentralización de la prestación de servicios de la SS hacia los estados se inició a mediados de la década de los ochenta y continuó a lo largo de la década de los noventa. ${ }^{3}$ Este importante paso hizo posible enfocar la función rectora de la SS en la coordinación, regulación, monitoreo y evaluación. No obs- tante, este proceso debilitó los instrumentos que la SS podía movilizar de manera efectiva para dirigir el sistema y alinear los incentivos.

Uno de los resultados clave de la reforma de 2003 fue fortalecer la función rectora de la SS a través de la generación de instrumentos para orientar los flujos financieros y vincular las asignaciones del lado de la oferta con los incentivos del lado de la demanda. Estos instrumentos incluyen reglas explícitas para las transferencias financieras del nivel federal al nivel estatal, el establecimiento de prioridades a través de un paquete de servicios esenciales y la certificación de la infraestructura de salud. Los incrementos presupuestales asociados con la reforma se manejan con estas herramientas para realinear los incentivos y, en consecuencia, corregir muchos de los desequilibrios financieros arriba descritos. Dichos instrumentos se exponen en esta serie en los trabajos de González-Pier y colaboradores, y Lozano y colaboradores., ${ }^{9,22}$

Los resultados de dos décadas de inversión para generar evidencia fueron clave para fortalecer la capacidad rectora de la SS, así como para orientar la reforma. La consolidación de instituciones públicas, como el Instituto Nacional de Salud Pública, y la creación de instancias no gubernamentales, como Funsalud, aproximadamente 20 años antes de la reforma, permitieron establecer una base institucional a partir de la cual fue posible generar encuestas, realizar análisis y capacitar a los formuladores de políticas.

El papel mismo de las evidencias como guía de las políticas se fortaleció como parte de la reforma. Por ejemplo, la reforma exigió la creación de un sistema de información integral sobre las familias afiliadas al Seguro Popular. Este sistema es operado por la SS y se utiliza como padrón para identificar el nivel de aportaciones de cada familia, con el fin de garantizar la transparencia en la asignación de recursos, y como herramienta gerencial que proporciona información sobre la utilización de servicios y sus resultados.

La reforma también está expandiendo la naturaleza y el horizonte del monitoreo y la evaluación. Desde 2001 se han publicado anualmente informes comparativos que hacen uso de indicadores del desempeño en los niveles sistémico, estatal, institucional y hospitalario. ${ }^{14,23-26}$ Estos indicadores utilizan las técnicas más avanzadas de que se dispone actualmente, junto con datos de encuestas a profundidad diseñadas específicamente para el sistema de salud mexicano. El informe de indicadores al nivel estatal ofrecerá un punto de referencia para la población sobre el funcionamiento del sistema y presionará a las instituciones para desempenarse mejor. 
La reforma misma está siendo sometida a una evaluación externa, rigurosa y de largo plazo, utilizando un diseño experimental que medirá con detalle el impacto del Seguro Popular sobre las condiciones de salud, la cobertura efectiva, la capacidad de respuesta del sistema de salud y la protección financiera. Con este fin, desde 2005 se han llevado a cabo encuestas longitudinales, diseñadas específicamente para medir los avances de la reforma. El primer conjunto de resultados se dará a conocer a finales de 2006. Los trabajos de Gakidou y colaboradores y Lozano y colaboradores, incluidos en esta serie, utilizan estos datos y presentan resultados que forman parte del proceso de evaluación. ${ }^{16,22}$

Diversos proyectos y programas sustantivos e instrumentales para mejorar la cobertura, la equidad y la calidad de los servicios de salud se están alineando como parte de la creación del Seguro Popular. Un ejemplo es la Cruzada Nacional por la Calidad de los Servicios de Salud lanzada en 2001 para mejorar la calidad técnica e interpersonal de la atención médica. Esto incluye la definición de los derechos de los pacientes y la introducción de mecanismos para la presentación tanto de quejas como de sugerencias sobre cómo mejorar los servicios.

Históricamente, las dependencias de salud del sector público han operado como monopolios que cuentan con un margen muy reducido para la elección del prestador del servicio, y muestran poco interés por satisfacer las expectativas del consumidor o mejorar la calidad de los servicios. Asimismo, pocos centros públicos o privados estaban sujetos a un proceso formal de acreditación. En este sentido, la cruzada representa una ruptura con el pasado. Está diseñada para resolver estos problemas concentrándose en mejorar los estándares de calidad de la atención, al tiempo que fortalece la capacidad de los ciudadanos para demandar la rendición de cuentas. El impulso para promover la calidad incluye un proceso de acreditación, reforzado por el hecho de que sólo los prestadores de servicios acreditados pueden participar en el Seguro Popular. Adicionalmente, se han diseñado e implantado indicadores para monitorear la calidad. Estos incluyen los tiempos de espera en hospitales y clínicas, así como indicadores sobre la distribución y surtimiento de medicamentos. Asimismo, en la mayoría de los estados se han establecido comisiones de arbitraje, con el fin de responder oportunamente a la expansión de las demandas por mala práctica médica. ${ }^{27}$

Algunas instituciones específicas dentro del sector salud también se han fortalecido para poder desempeñar mejor su función rectora. En algunos casos esto significó definir nuevas funciones para entidades ya existentes; en otros implicó desarrollar nuevas enti- dades. Uno de los ejemplos más importantes es el Consejo de Salubridad General, una antigua institución creada en 1910 como organismo formulador de políticas del más alto nivel dentro del sector salud. Encabezado por el Secretario de Salud, el Consejo incluye a los titulares de todas las instituciones públicas de salud del país, a expertos destacados en el campo de la salud, a organizaciones civiles y asociaciones profesionales, y al sector privado. Actualmente el Consejo es responsable de un elemento clave del proceso de definición de prioridades: la definición de las enfermedades y, por ende, los tratamientos y medicamentos correspondientes que habrán de ser considerados para cobertura por el Fondo de Protección contra Gastos Catastróficos.

En el ámbito de la regulación, en 2001 se creó la Comisión Federal para la Protección contra Riesgos Sanitarios. Esta dependencia reguladora semiautónoma opera de manera independiente de la SS con un enfoque tanto interinstitucional (dentro del sector salud) como intersectorial, para lograr una mejor coordinación. La Comisión reglamenta productos y servicios críticos, como los productos farmacéuticos y las tecnologías de salud, los riesgos ocupacionales y ambientales, el saneamiento básico, la inocuidad alimentaria y la publicidad relacionada con la salud. Es de particular importancia su función en la regulación e inspección de hospitales y clínicas, ya que está obligada a clausurar a los prestadores de servicios que no cumplan con las normas básicas de calidad.

\section{Financiamiento}

La lógica que subyace a la reforma es separar el financiamiento entre los bienes públicos de la salud y los servicios personales de salud (cuadro I). Esta separación del financiamiento está diseñada para proteger las actividades de salud pública, que tienden a estar subfinanciadas en las reformas que se sustentan en subsidios a la demanda para los servicios personales de salud. ${ }^{28}$ Los bienes públicos de salud incluyen la función de rectoría (planeación estratégica, información, evaluación, investigación y desarrollo de recursos humanos), así como los servicios de salud comunitaria. Las funciones de rectoría se financian a través del presupuesto de la SS. El nuevo Fondo de Aportaciones para los Servicios de Salud a la Comunidad se usa para financiar exclusivamente las actividades de salud pública proporcionadas en los niveles federal y estatal. ${ }^{2,29}$

El financiamiento de los servicios personales de salud se basa en una lógica de aseguramiento que permite proteger a los pacientes contra la incertidumbre financiera asociada con la enfermedad. El Seguro Popu- 
lar divide los servicios personales de salud en un paquete esencial de intervenciones de primer y segundo nivel, que se proporcionan en el contexto de la atención ambulatoria y en hospitales generales, y un paquete de intervenciones de alto costo de tercer nivel, financiadas a través del Fondo de Protección contra Gastos Catastróficos (FPGC). La prestación del paquete esencial de intervenciones se ha descentralizado al nivel estatal, ya que se trata de intervenciones que se asocian con eventos de salud de bajo riesgo y alta probabilidad. En contraste, las intervenciones de alto costo de tercer nivel requieren de un fondo que mancomune el riesgo al nivel nacional, así como de unidades regionales o nacionales que ofrezcan servicios de alta especialidad financiados por el FPGC. 2,9,29

El Seguro Popular ofrece protección financiera en materia de salud a los mexicanos que no cuentan con ningún tipo de seguro público de salud. Su estruc- tura financiera se basa en una lógica tripartita de derechos y responsabilidades que es similar a la de las dos dependencias de seguridad social más importantes -IMSS e ISSSTE (cuadro II)-. Esta estructura garantiza solidaridad y corresponsabilidad entre los diferentes órdenes de gobierno y las familias. La estructura financiera tripartita del Seguro Popular incluye aportaciones de tres fuentes: el gobierno federal, un contribuyente corresponsable y el beneficiario.,29

El primer componente es la contribución social o cuota social, la cual es una aportación fija por familia financiada totalmente por el gobierno federal con ajustes periódicos de acuerdo a la inflación. Se basa en el principio ético del derecho universal de acceso a los servicios de salud y por lo tanto es igual para todas las familias mexicanas. Esta contribución garantiza la distribución equitativa de los recursos federales y, en consecuencia, la solidaridad entre todos los grupos po-

Cuadro I

Relación entre tipos de bienes y fondos en el Sistema de Protección Social en Salud, México

\begin{tabular}{|c|c|c|}
\hline Tipo de bien & Bienes de la salud & Fondo \\
\hline \multirow{3}{*}{ Bienes públicos de salud } & Rectoría & \multirow{2}{*}{ Presupuesto de la Secretaría de Salud } \\
\hline & $\begin{array}{l}\text { Información, evaluación, investigación, desarrollo } \\
\text { de recursos humanos }\end{array}$ & \\
\hline & Servicios de salud a la comunidad & $\begin{array}{l}\text { Fondo de Aportaciones para los } \\
\text { Servicios de Salud a la Comunidad }\end{array}$ \\
\hline \multirow{2}{*}{$\begin{array}{l}\text { Servicios personales de } \\
\text { salud } \\
\text { (Seguro Popular) }\end{array}$} & $\begin{array}{l}\text { Servicios esenciales de salud } \\
\text { (atención de primer y segundo nivel) }\end{array}$ & $\begin{array}{l}\text { Fondo para Servicios Personales de } \\
\text { Salud }\end{array}$ \\
\hline & $\begin{array}{l}\text { Servicios de salud de tercer nivel de alta especialización } \\
\text { asociados con gastos catastróficos }\end{array}$ & $\begin{array}{l}\text { Fondo de Protección Contra Gastos } \\
\text { Catastróficos }\end{array}$ \\
\hline
\end{tabular}

Cuadro II

Contribuciones para financiar la protección social universal en salud, México

Esquema de aseguramiento público

IMSS (empleados asalariados del sector privado)

ISSSTE (empleados asalariados del sector público)

Seguro Popular (empleados no asalariados, trabajadores independientes, $y$ familias fuera de la fuerza de trabajo)

\begin{tabular}{|l|l|l|}
\hline \multicolumn{2}{c}{ Contribuciones } \\
\hline Beneficiario & Contribuyente corresponsable & Gobierno Federal \\
\hline Empleado & Empleador privado & Contribución social \\
\hline Empleado & Empleador público & Contribución social* $^{*}$ \\
\hline Familia & $\begin{array}{l}\text { Contribución solidaria: } \\
\text { Gobierno estatal/Gobierno federal }\end{array}$ & Contribución social \\
\hline
\end{tabular}

* En el caso del ISSSTE, la contribución social todavía no se establece, ya que los cambios legislativos que se requieren están en desarrollo 
blacionales dado que los fondos federales provienen de los impuestos generales.

El segundo elemento es la contribución corresponsable, que garantiza la solidaridad dentro de cada grupo poblacional y la redistribución entre estados. En el IMSS esta aportación proviene del patrón y en el ISSSTE del gobierno en su calidad de empleador. En el caso del Seguro Popular, dado que no hay empleador, la corresponsabilidad se establece entre los gobiernos federal y estatal para subsanar las enormes diferencias entre los niveles de desarrollo de los estados. La aportación solidaria federal es, en promedio, 1.5 veces la contribución social, pero se incrementa para los estados más pobres a expensas de los más ricos. La aportación solidaria estatal es igual para todos los estados y asciende a la mitad de la contribución social federal. La fuente de financiamiento son los recursos provenientes de los ingresos estatales. ${ }^{2,29}$

El tercer componente es la contribución familiar, que es progresiva y redistribuye el ingreso familiar. En el caso del IMSS y el ISSSTE, la aportación del empleado se deduce de la nómina. En el Seguro Popular el monto de la aportación se basa en un subsidio escalonado con base en el principio de que ninguna familia debería tener que aportar una cantidad mayor a la proporción justa en función de su capacidad de pago. Para promover la justicia financiera, la capacidad de pago se define en términos del ingreso disponible que, a su vez, se define como el gasto total del hogar menos el gasto en alimentos. Las familias de los dos deciles de ingresos más bajos no realizan aportaciones financieras, pero su afiliación está condicionada a su participación en actividades de promoción de la salud. Para los demás deciles de ingreso, la aportación de la familia es una proporción fija e igual del ingreso disponible y el límite superior es 5\%. Para cada decil del tercero al noveno se definió una aportación nominal, y se establecieron dos niveles de aportación para el décimo decil debido a la amplia variación en la parte superior de la distribución del ingreso.

Este esquema financiero incluye varios fondos etiquetados. El más grande de ellos es el FPGC, que equivale a $8 \%$ de la contribución social federal más las aportaciones solidarias federal y estatal. Un $2 \%$ adicional está dirigido a las inversiones en infraestructura en comunidades pobres. Un fondo de reserva que equivale a $1 \%$ del total está diseñado para cubrir las fluctuaciones inesperadas de la demanda y los pagos temporalmente vencidos de la utilización interestatal de servicios. Estos tres fondos se administran a nivel federal para garantizar la adecuada mancomunación de riesgos. El resto de las aportaciones se asigna a los estados para financiar el paquete esencial de servi- cios de salud. La totalidad de las contribuciones familiares permanece en el estado que las recolectó.

Este modelo de financiamiento implica un cambio importante en los incentivos para los gobiernos estatales y los prestadores de servicios. Ahora el financiamiento para los estados se basa en una fórmula y ésta, a su vez, está determinada en gran parte por el número de familias afiliadas al Seguro Popular y, por ende, por la demanda. En el pasado los presupuestos estatales de salud eran asignados por el gobierno federal y estaban determinados en gran parte por la inercia histórica y el tamaño de la nómina del sector salud. Como consecuencia de la reforma, la "presupuestación burocrática", orientada a satisfacer las demandas de los prestadores de servicios, está siendo gradualmente reemplazada por lo que se ha denominado "presupuestación democrática", que garantiza que el dinero siga a la gente. La fórmula también incluye un componente de desempeño y un componente de progresividad para compensar gradualmente las diferencias entre los estados. ${ }^{2,29}$

La afiliación es voluntaria. Sin embargo, la reforma incluye incentivos para ampliar la cobertura. Los estados tienen el incentivo de afiliar a toda la población porque su presupuesto se basa en un pago anual por familia. La naturaleza voluntaria de la afiliación es un componente clave del proceso, ya que genera incentivos para equilibrar la calidad y la eficiencia: si los estados ofrecen una atención dispendiosa, el dinero recibido no será suficiente para satisfacer la demanda, pero si la calidad es baja, las familias no se reafiliarán.,2,

\section{Prestación de servicios}

Los servicios para los afiliados al Seguro Popular se contratan en su mayor parte, pero no exclusivamente, con prestadores públicos que predominantemente forman parte de las 32 secretarías estatales de salud. Se han diseñado varias iniciativas para fortalecer la oferta de servicios de salud de alta calidad. Dentro de ellas destacan los tres planes maestros de infraestructura, equipamiento y recursos humanos. ${ }^{29}$

En el caso de la infraestructura, el plan maestro fue diseñado para proporcionar un marco de largo plazo para ampliar la disponibilidad y capacidad de las unidades que ofrecen servicios de salud. Antes de la reforma había pocos incentivos para invertir en infraestructura y el financiamiento disponible era escaso. La reforma contrarresta este problema mediante el aumento de la inversión pública en servicios de salud durante siete años. Dicho aumento está alineado con la afiliación, ya que los estados tienen el incentivo de atender a la población afiliada al Seguro Popular en 
tanto su presupuesto está ligado a los niveles de afiliación. En vista de que las etapas iniciales de la afiliación se enfocaron en los segmentos más pobres de la población, las prioridades en términos de las inversiones de capital se relacionan con esas familias y, en consecuencia, son altamente progresivas. Para maximizar la eficiencia, el financiamiento para las unidades nuevas -clínicas, centros de salud, hospitales de segundo nivel y hospitales de especialidades de tercer nivel-se basa en la determinación de necesidades realizada por el estado en cada localidad. Para 2006 se habrán construido 1792 nuevas unidades de salud, que incluyen cuatro hospitales regionales de alta especialidad en la región sur, la menos desarrollada del país.

Un complemento del plan maestro de infraestructura es el plan de inversión en equipamiento médico, diseñado e implementado por el Centro Nacional de Excelencia Tecnológica en Salud (Cenetec), de reciente creación. Este plan refuerza los procedimientos de mantenimiento del equipo médico y racionaliza la adopción de nuevas tecnologías a través de la valoración de necesidades basadas en evidencias.

Finalmente, el plan maestro de inversión en recursos humanos se enfoca en el desarrollo de las capacidades médicas y de enfermería necesarias para satisfacer las cambiantes demandas asociadas con la transición epidemiológica y para equilibrar un mercado de trabajo médico que presenta, al mismo tiempo, problemas de desempleo urbano y escasez de dichos recursos en las áreas rurales. ${ }^{30}$ Este plan incluye un diagnóstico exhaustivo por estado de la oferta de médicos, enfermeras y otros trabajadores de la salud en las instituciones públicas. A partir de esta información se hacen proyecciones de mediano plazo de la oferta y se emiten recomendaciones en materia de desarrollo de recursos humanos, tanto para las instituciones educativas como para los prestadores públicos de servicios.

Junto con los tres planes maestros se han desarrollado otras herramientas para mejorar la prestación de servicios. Destacan dentro de ellas las cartillas de salud diseñadas para facilitar la prevención y la detección temprana de enfermedades en grupos específicos de edad y sexo. Se ha puesto particular énfasis en las intervenciones relacionadas con las Metas de Desarrollo del Milenio, incluida la salud neonatal e infantil, como es analizada por Sepúlveda y colaboradores en otro artículo de esta serie. ${ }^{31,32}$ Asimismo, se han obtenido resultados importantes en la prevención y control del VIH/SIDA. Las tarjetas de salud han sido muy útiles para el manejo de enfermedades crónicas no transmisibles y factores de riesgo como la obesidad, diabetes, hipertensión y cáncer. Los programas de de- tección temprana de cáncer cervicouterino y cáncer de mama están teniendo resultados alentadores, como se menciona en Gakidou y colaboradores en esta serie. ${ }^{16}$

La herramienta clave que articula los incentivos del lado de la oferta y de la demanda es el paquete de servicios cubiertos. De acuerdo con la Ley, el paquete esencial debe incluir atención ambulatoria y hospitalaria para las especialidades básicas (medicina interna, cirugía general, ginecología y obstetricia, pediatría y geriatría). A partir de 2006, el paquete de servicios esenciales incluye 249 intervenciones, mientras que el FPGC financia 17 intervenciones más.

El proceso de definición del paquete es dinámico. La ley estipula que debe ampliarse progresivamente y actualizarse cada año con base en los cambios en el perfil epidemiológico, los avances tecnológicos y la disponibilidad de recursos. Esto significa que la cobertura de beneficios se amplía con el tiempo a medida que se cuenta con nuevas tecnologías y mayores recursos, y también conforme se identifican nuevas enfermedades. Los servicios cubiertos se analizan y eligen con base en las evidencias derivadas de estudios de costoefectividad y también con base en la deliberación ética de criterios de aceptabilidad social. El uso de este enfoque se ilustra en los trabajos de González-Pier y colaboradores, y Lozano y colaboradores en esta serie. 9,22

La noción de un paquete de servicios de salud, además de servir de herramienta para la definición de prioridades desde el punto de vista de la rectoría, es un medio para empoderar a las personas al hacer explícitos sus derechos. De igual modo, es un instrumento clave de planeación para orientar a los prestadores de servicios y una guía para la acreditación, puesto que define los servicios de salud que cada prestador debe ofrecer ahora y en el futuro. En otras palabras, la definición del paquete y la organización del proceso de acreditación de los prestadores en torno a él generan las condiciones para que el sistema realmente proporcione las intervenciones específicas que, de acuerdo con las evidencias disponibles, producen los mayores beneficios de salud dado un determinado nivel de recursos.

\section{Avances y retos futuros}

La reforma está produciendo resultados positivos: el monto de los recursos públicos dedicados a la salud ha aumentado significativamente; se espera que el número de familias aseguradas llegue a 5.1 millones para fines de 2006, de acuerdo con el mandato legal de afiliar cada año a 14\% de la población no asegurada, y el conjunto de servicios médicos a los que cada familia con cobertura tiene acceso se ha ampliado de manera considerable. 


\section{Evolución de los desequilibrios financieros}

El gasto total en salud en México, expresado como porcentaje del PIB, creció de $6.2 \%$ en 2002 a $6.5 \%$ en 2006 (cuadro III). De hecho, entre 2001 y 2006 el presupuesto de la SS aumentó $69 \%$ en términos reales. La mayor parte de este aumento es consecuencia directa de la movilización de recursos públicos adicionales vinculados con la reforma. Sobre esta base se espera que el gasto público en salud siga aumentando a un ritmo mayor que el gasto privado. Así, se espera que para 2010 el sistema mexicano de salud esté financiado, en su mayor parte, con fondos públicos. Esto se basa en los resultados presentados por Knaul y colaboradores en esta serie, que muestran la constante reducción de los gastos catastróficos y empobrecedores en salud desde la crisis económica de 1994-1996. ${ }^{13}$ Aun cuando parte de esta mejoría se atribuye a la recuperación económica y al combate a la pobreza por medio de programas sociales como Oportunidades, existe evidencia descriptiva sustancial que sugiere que también se asocia con la expansión del Seguro Popular y las inversiones concomitantes para fortalecer la prestación de servicios. ${ }^{1}$

El aumento del financiamiento público también está cerrando la brecha en términos de las asignaciones per cápita entre la población sin seguridad social (incluidos los afiliados al Seguro Popular) y la población cubierta por la seguridad social. En el periodo 2002-2006 la rela- ción entre el gasto público per cápita entre ambos grupos disminuyó de 2.3 a 2.0 y continuará declinando con la expansión legislada del Seguro Popular.

Las inequidades en la distribución de los recursos públicos entre los estados también se han reducido. En los últimos cuatro años la diferencia en las asignaciones federales per cápita entre el estado que recibió la mayor asignación y el que recibió la menor disminuyó de 5 a 4 veces. En el mismo periodo, la desigualdad en las contribuciones estatales para financiar la salud también disminuyó, como lo muestra el coeficiente de variación, que se redujo ligeramente, de 1.14 en 2002 a 1.11. Finalmente, la proporción de recursos públicos asignados a la inversión en infraestructura se incrementó.

\section{Cobertura}

Este nuevo escenario financiero ha hecho posible aumentar la cobertura del Seguro Popular en dos dimensiones: horizontal y vertical. La primera se relaciona con el número de familias afiliadas; la segunda se refiere a los beneficios que cubre (figura 2). Estos últimos se analizan en González-Pier y colaboradores en esta serie. ${ }^{9}$

La cobertura horizontal se está expandiendo mediante la aplicación de criterios para promover la equidad y la justicia, ya que la afiliación se inició entre los grupos más pobres de la población y se está extendiendo gradualmente hacia los de mayores ingresos. La ley define el plan de cobertura horizontal. Para 2010,

Cuadro III

Evolución de los desequilibrios financieros QUe exigían UNA Reforma INTEgral en MéXico

\begin{tabular}{|c|c|c|c|}
\hline & Indicador & 2002 & Avance \\
\hline Nivel & Gasto en salud como \% del PIB & $6.2 \%$ & $\begin{array}{c}6.5 \% \\
\left(2006^{\ddagger}\right)\end{array}$ \\
\hline Fuente & Gasto público en salud como \% del gasto total en salud & $43.9 \%$ & $\begin{array}{l}46.4 \% \\
\left(2006^{\ddagger}\right)\end{array}$ \\
\hline \multirow[t]{2}{*}{ Distribución } & $\begin{array}{l}\text { Número de veces que el gasto público per cápita para la población } \\
\text { asegurada excede el gasto público per cápita para la población no asegurada* }\end{array}$ & 2.3 & $\begin{array}{c}2.0 \\
\left(2006^{\ddagger}\right)\end{array}$ \\
\hline & $\begin{array}{l}\text { Relación entre el gasto federal per cápita en salud en el estado } \\
\text { con la cifra más alta y el estado con la cifra más baja }\end{array}$ & 5 a I & $\begin{array}{l}4 \mathrm{a} \mathrm{l} \\
(2004)\end{array}$ \\
\hline Contribución estatal & $\begin{array}{l}\text { Variabilidad en la contribución de los estados al financiamiento } \\
\text { de los servicios de salud (coeficiente de variación) }\end{array}$ & 1.14 & $\begin{array}{c}1.11 \\
(2004)\end{array}$ \\
\hline Distribución de recursos & Relación entre el gasto en nómina y la inversión en infraestructura & 59 a I & $\begin{array}{c}7 \mathrm{a} \mathrm{I} \\
\left(2006^{\ddagger}\right)\end{array}$ \\
\hline
\end{tabular}


$100 \%$ de la población actualmente no asegurada (12 millones de familias) deberá estar afiliada. Por lo tanto, aproximadamente 1.7 millones de familias adicionales deben ser cubiertas cada año. Entre 2001 y 2003, antes de la reforma legal, el Seguro Popular operaba como un programa piloto que afilió a 614000 familias. Desde entonces, la expansión de la cobertura se ha dado según lo programado y para septiembre de 2006 se habrán afiliado cuatro millones de familias -el equivalente a casi 15 millones de personas-. Esto significa que el Seguro Popular ya es el segundo seguro de salud más grande del país. La mayoría de las familias afiliadas pertenecen a los dos deciles de ingresos más bajos $y$, en consecuencia, no se les exige el pago de una contribución familiar.

La expansión geográfica se ha dado en función del momento en que cada estado introdujo el nuevo sistema, la rapidez con que cada estado ha podido cubrir a los determinados grupos poblacionales y la disponibilidad de recursos adicionales. Desde principios de 2005 los 32 estados de México participan en el Seguro Popular. En términos de progresividad, en el año 2000 sólo 7\% de las familias del quintil más pobre estaban aseguradas (primordialmente a través del IMSS o ISSSTE). Para 2004 esta cifra había aumentado a $37 \%$ a medida que las familias de este grupo se afiliaron al Seguro Popular., ${ }^{1,26}$

La cobertura vertical se está ampliando con base en prioridades explícitas en materia de intervenciones, como se indicó anteriormente. Los servicios actual- mente cubiertos representan una expansión significativa respecto a la situación previa a la reforma y un avance constante a partir de 2003. Actualmente, el paquete de servicios esenciales cubre aproximadamente 95\% de todas las causas de hospitalización, y 17 intervenciones de alta complejidad son cubiertas a través del FPGC. Este último también prevé un esquema especial de cobertura universal acelerada para problemas de alta prioridad considerados como las principales causas de gasto catastrófico para las familias previamente no aseguradas, incluyendo terapia integral de SIDA, tratamiento de cánceres infantiles, tratamiento de cáncer cervicouterino en todas sus fases y extracción de cataratas. Mediante el esquema de cobertura acelerada, toda persona no asegurada que requiera de los servicios cubiertos es susceptible de afiliación inmediata al Seguro Popular. Las intervenciones cubiertas a través de los esquemas gradual y acelerado se ampliarán continuamente a medida que los recursos aumenten conforme se incremente el número de familias afiliadas.

\section{Retos futuros}

Aunque la reforma ha logrado avances, aún persisten varios retos. En primer lugar, se requieren recursos públicos adicionales tanto para continuar remplazando el gasto del bolsillo, como para satisfacer la costosa demanda asociada con la transición epidemiológica, especialmente de intervenciones hospitalarias de alta

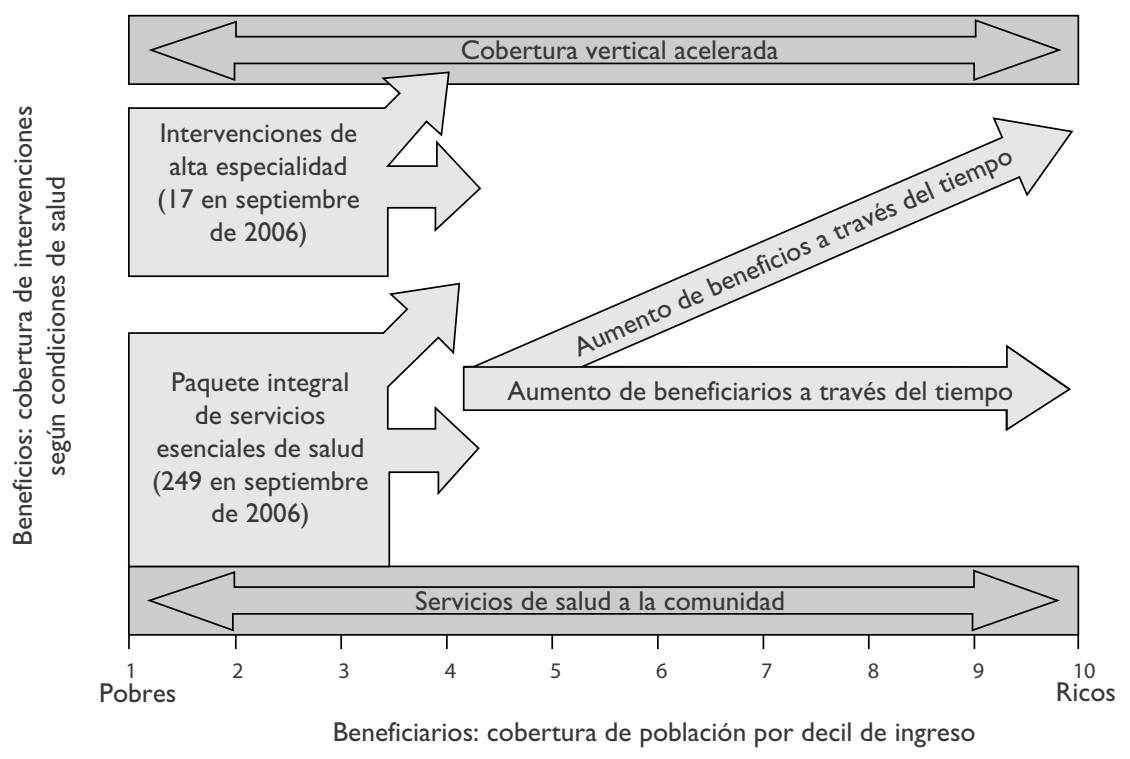

Figura 2. Estrategias de la cobertura horizontal y vertical en el sistema de protección social en MéXico 
complejidad. Esto comenzará a cerrar la brecha entre México y otros países de la OCDE y la región latinoamericana, en los cuales una mayor proporción del gasto en salud se financia con recursos públicos. ${ }^{6}$ Aunque algunos recursos serán liberados con el aumento de la eficiencia resultado de los incentivos integrados en la reforma, el monto absoluto y la proporción del PIB dedicada a salud proveniente de recursos públicos debe aumentar para que el sistema de salud responda efectivamente a las demandas de la población, alcance la cobertura universal del aseguramiento en salud, y expanda el número de intervenciones a las que tienen derecho las familias afiliadas. También será necesario continuar mejorando la distribución de los recursos públicos entre poblaciones y estados, y garantizar un compromiso presupuestal sostenido por parte de las autoridades locales. Otro reto en materia financiera será alcanzar el equilibrio correcto entre las inversiones adicionales en promoción de la salud y prevención de enfermedades, por un lado, y en servicios personales de salud, por el otro.

Además de una mayor cantidad de recursos, la persistente fragmentación institucional todavía representa un reto para promover una estructura general de financiamiento del sistema mexicano de salud más equitativa. Desde esta perspectiva, se requieren nuevos pasos que promuevan la integración de las fuentes públicas de financiamiento a través de los esquemas de protección social existentes, con el fin de garantizar una mancomunación adecuada de los riesgos en el ámbito nacional, especialmente para la atención altamente especializada de tercer nivel.

Aumentar la orientación al cliente y la capacidad de respuesta del sistema de salud constituyen un tercer reto, especialmente considerando las crecientes expectativas, tanto de pacientes como de prestadores de servicios, generadas por el proceso de democratización del país y por la reforma misma. Para reafiliarse al Seguro Popular y estar dispuestos a hacer una contribución financiera anticipada, los ciudadanos deben sentir mayor confianza en los servicios públicos, y esto sólo puede lograrse si el sistema de salud es capaz de satisfacer efectivamente sus necesidades de salud. Para lograr esta meta es necesario seguir mejorando las siguientes áreas: la calidad técnica de la atención, la disponibilidad de medicamentos, la capacidad de elección del prestador de atención médica, el acceso a la atención durante las noches y fines de semana, y los tiempos de espera tanto para la atención ambulatoria y de emergencia, como para las intervenciones electivas.

Otro reto es desarrollar un ambiente más competitivo entre los prestadores de servicios. Muy probablemente los intereses creados relacionados con el poder monopólico en la prestación de servicios de salud dentro del sector público se verán amenazados con la nueva ley, la cual incrementa la capacidad de elección de los pacientes. Sin embargo, en el largo plazo este ambiente competitivo ayudará a alcanzar el equilibrio correcto entre calidad y eficiencia.

Finalmente, uno de los principales desafíos de la reforma mexicana será extender las innovaciones adoptadas por la SS hacia las instituciones de seguridad social. Esto incluye la separación efectiva entre el comprador y el prestador de servicios, y el incremento de la capacidad de elección de los pacientes. A su vez, las innovaciones recientes en la organización de los prestadores y las mejoras en las prácticas administrativas introducidas por el IMSS deben extenderse al resto del sistema. La homologación de las reglas de financiamiento y la adopción de estándares de calidad similares en todas las dependencias públicas podría gradualmente derribar los obstáculos institucionales existentes y abrir la puerta a un sistema más eficiente.

\section{Lecciones}

La experiencia de la reforma mexicana de salud ofrece un conjunto de lecciones potencialmente relevantes para otros países que están considerando realizar reformas de la salud en escenarios de desafío similares. La mayoría de los países en desarrollo enfrentan la apremiante combinación de una rápida transición epidemiológica con un sistema de salud subfinanciado y abrumado que es incapaz de responder a las circunstancias. En este contexto, es necesario diseñar e implementar propuestas de reforma integrales basadas en los tres pilares de las políticas públicas: ético, técnico y político. ${ }^{18,33}$

Por lo que se refiere al primer pilar, la reforma mexicana se formuló y promovió sobre la base de un conjunto de valores y principios explícitos relacionados con la idea de que la atención de la salud no es una mercancía o un privilegio, sino un derecho social. El ejercicio de este derecho puede mejorar las condiciones de salud de la población, el capital humano de las personas y los hogares, y la situación de pobreza del país. Este razonamiento resulta particularmente atractivo dado el proceso de democratización que experimentan muchos países en desarrollo y economías en transición. ${ }^{34}$

El pilar técnico es producto de una inversión de largo plazo en el desarrollo institucional y de recursos humanos, y el uso de bienes públicos globales relacionados con el conocimiento que se adaptaron al contexto mexicano. Entre estos bienes se incluyen los marcos 
conceptuales (por ejemplo, el marco de la OMS para el Desempeño de los Sistemas de Salud), los métodos estandarizados (por ejemplo, las encuestas de ingresos y gastos de hogares), y las herramientas analíticas (por ejemplo, las cuentas nacionales de salud). La inversión en recursos humanos durante un lapso de dos décadas hizo posible adaptar los conocimientos generados en todo el mundo y recabar evidencias que apoyaran una transformación profunda del sistema de salud. Estas evidencias empoderaron a los formuladores de políticas mexicanos en sus discusiones tanto con la Secretaría de Hacienda como con el Congreso, y guiaron el diseño, implementación y evaluación de la reforma. ${ }^{1}$

El pilar político se refiere al desarrollo de consensos para lograr objetivos compartidos. La reforma mexicana se benefició del concepto de que la salud es una aspiración de todas las fuerzas políticas y, por ende, puede generar acuerdos amplios y ayudar a incrementar la cohesión social. El énfasis en principios democráticos -transparencia, rendición de cuentas y empoderamiento de los ciudadanos- también ayudó a generar el apoyo requerido y a consolidar la muy necesaria participación pública en todos los problemas relacionados con la atención de la salud. En términos del proceso político, resultó esencial la participación temprana de los actores clave en la discusión de la reforma, una vez que se definieron los cambios necesarios y se identificaron las opciones de política. Esto significó llevar a cabo campañas intensivas de consulta en todo el país para hacer conciencia y debatir sobre la reforma. Siempre que fue posible, se buscó conciliar los intereses de los sectores público y privado; de las autoridades federales, estatales y locales, así como de los grupos de interés, los sindicatos, los legisladores y los formuladores de política, para alcanzar cierto grado de consenso alrededor de un tema tan sensible como la salud. Finalmente, la estrategia de introducción de la reforma se organizó bajo un enfoque gradual. Esto dio el tiempo necesario para generar aceptación política y para desarrollar la capacidad de respuesta de la oferta de servicios a nivel local. La implementación del Seguro Popular se ha sustentado en la priorización de grupos de población, estados y localidades para generar un equilibrio justo entre la rapidez, percepción y progresividad de la respuesta de la oferta que se ha enfocado en los pobres. Más aún, la extensión del Seguro Popular se ha realizado utilizando una selección de municipios y estados para atender a los más pobres, pero también para hacerlo sin sesgo político alguno. Como resultado del esfuerzo significativo para aumentar el grado de conciencia mediante el uso de evidencias, la reforma ha logrado ser reconocida como un objetivo compartido por todas las partes involucradas y en todas las regiones. Esto ha facilitado en gran medida la negociación anual con el Congreso para buscar las asignaciones presupuestales necesarias cada año a fin de lograr las metas de afiliación.

La evidencia, como aquella presentada en cada uno de los trabajos de esta serie, fue un elemento crucial tanto para desarrollar el modelo -el trabajo técnico-, como para promover la legislación y obtener el financiamiento -el trabajo político-. Así, el uso de las evidencias continuará siendo un elemento esencial para mantener el ímpetu de la reforma. En ese sentido, la experiencia mexicana ofrece un modelo conceptual y práctico que busca alcanzar el objetivo tan evasivo de la cobertura universal, de manera que cada persona tenga igual oportunidad para ejercer su derecho a una atención médica de alta calidad con protección social para toda la población.

\section{Agradecimientos}

Agradecemos a Mariana Barraza-Lloréns, María Lilia Bravo, Emmanuela Gakidou, Cristina GutiérrezDelgado, Rachel Maguire, Héctor Peña-Baca, Maja Pleic, Gloria Saldaña, Tania Martínez, Karina Rincón y Armando Vargas-Palacios, por sus valiosos comentarios y ayuda con diversos aspectos de este trabajo. Felicia Knaul recibió apoyo del Centro Internacional de Investigaciones para el Desarrollo (IDRC, por sus siglas en inglés) de Canadá, así como del Consejo Nacional de Ciencia y Tecnología (Conacyt) de México y del Consejo Promotor Competitividad y Salud de la Fundación Mexicana para la Salud.

\section{Referencias}

I. Frenk J.Tender puentes: lecciones globales desde México sobre políticas de salud basadas en evidencias. Salud Publica Mex 2007;49 supl $\mathrm{I}: \mathrm{SI}$ 4-S22.

2. Knaul F, Frenk J. Health insurance in Mexico: achieving universal coverage through structural reform. Health Aff 2005;24(6): I 467-I 476. 3. Frenk J, Sepúlveda J, Gómez-Dantés O, Knaul F. Evidence-based health policy: three generations of reform in Mexico. Lancet 2003;362:I667-I67I. 4. Londoño JL, Frenk J. Structured pluralism: towards a new model for health systems reform in Latin America. Washington, DC:World Bank, 1995.

5. Barraza-Lloréns M, Bertozzi S, González-Pier E, Gutiérrez JP. Addressing inequity in health and health care in Mexico. Health Aff 2002;2I(3):47-56.

6. Organization for Economic Cooperation and Development. OECD reviews of health systems, México. Paris: OECD, 2005.

7. Instituto Nacional de Estadística, Geografía e Informática (INEGI), Indicadores Sociodemográficos de México (1930-2000). http:// www.inegi.gob.mx/prod_serv/contenidos/espanol/bvinegi/productos/ 
integracion/sociodemografico/indisociodem/200I/indi200I.pdf [consultado en: 29 de agosto de 2006].

8. Consejo Nacional de Población. Proyecciones de Población de México 2000-2050. Conapo. http://www.conapo.gob.mx/00cifras//5.htm [consultado en: 29 de agosto de 2006].

9. González-Pier E, Gutiérrez-Delgado C, Stevens G, Barraza-Lloréns M, Porras-Condey R, Carvalho $\mathrm{N}$ et al. Definición de prioridades para las intervenciones de salud en el Sistema de Protección Social en Salud de México. Salud Publica Mex 2007;49 supl I:S37-S52.

10. Frenk J, Bobadilla JL, Sepúlveda J, López-Cervantes M. Health transition in middle-income countries: new challenges for health care. Health Policy Plan 1989;4:29-39.

II. Secretaría de Salud. Programa Nacional de Salud 200 I-2006. La democratización de la salud en México. Hacia un sistema universal de salud. México, DF: Secretaría de Salud, 2001.

12. Frenk J, Knaul F, Gómez-Dantés $O$ et al. Fair financing and universal protection: the structural reform of the Mexican health system. México, DF: Secretaría de Salud, 2004

13. Knaul FM, Arreola-Ornelas H, Mendez-Carniado O, Bryson-Cahn C, Barofsky J, Maguire R, et al. Las evidencias benefician al sistema de salud: reforma para remediar el gasto catastrófico y empobrecedor en salud en México. Salud Publica Mex 2007;49 supl I:S70-S87.

14. Secretaría de Salud. Salud: México, 200I. Información para la rendición de cuentas. México, DF: Secretaría de Salud, 2002.

15. Knaul FM, Arreola-Ornelas H, Méndez O, Miranda M. Preventing impoverishment, promoting equity and protecting households from financial crisis: universal health insurance through institutional reform in Mexico.Working paper. México, DF: Fundación Mexicana para la Salud, 2005. Disponible en: http://www.gdnet.org/pdf2/gdn_library/ awards_medals/2005/medals_cat3_first.pdf [consultado en: 18 de septiembre de 2006].

16. Gakidou E, Lozano R, González-Pier E, et al.Assessing the effect of the 200I-2006 Mexican Health Reform: an interim report card. Lancet 2006;368:1920-1935.

17. Knaul F,Arreola H, Méndez O. Protección financiera en salud, México 1992 a 2004. Salud Publica Mex 2005;47:430-439.

18. Frenk J, Knaul FM, Gómez-Dantés O. Closing the relevanceexcellence gap in health research: the use of evidence in Mexican health reform. En: Global Forum update on research for health. London: ProBrook Publishing, 2004:48-53.
19. World Health Organization. World Health Report 2000. Health systems: improving performance. Ginebra:WHO, 2000.

20. Frenk J. Dimensions of health system reform. Health Policy 1994;27:19-34.

21. Murray CJL, Frenk J.A framework for assessing the performance of health systems. Bull World Health Organ 2000;78(6):717-73I.

22. Lozano R, Solis P, Gakidou E, et al. Benchmarking performance of mexican states using effective coverage. Lancet 2006;368: I729- I74I. 23. Secretaría de Salud. Salud: México 2002. Información para la rendición de cuentas. México, DF: Secretaría de Salud, 2003. 24. Secretaría de Salud. Salud: México 2003. Información para la rendición de cuentas. México, DF: Secretaría de Salud, 2004.

25. Secretaría de Salud. Salud: México 2004. Información para la rendición de cuentas. México, DF: Secretaría de Salud, 2005.

26. Secretaría de Salud. Salud: México 200I-2005. Información para la rendición de cuentas. México, DF: Secretaría de Salud, 2006. 27. Tena C, Sotelo J. Malpractice in Mexico: arbitration not litigation. BM] 2005;33I:448-45I.

28. Restrepo JH. Diseño y experiencia de la regulación en salud en Colombia. Revista de Economía Institucional 2005;7:165-190.

29. Secretaría de Salud. Sistema de Protección Social en Salud. Elementos conceptuales, financieros y operativos. México, DF: Secretaría de Salud, 2005.

30. Frenk J, Knaul F, Vázquez-Segovia LA, Nigenda G.Trends in medical employment: persistent imbalances in urban Mexico. Am J Public Health 1999;89:1054-1058.

31 . Horton R. The coming decade for global action on child health Lancet 2006;367:3-5.

32. Sepúlveda J, Bustreo F, Tapia R, Rivera J, Lozano R, Olaiz G et al. Aumento de la sobrevida en menores de cinco años en México: la estrategia diagonal. Salud Publica Mex 2007;49 supl I:SII0-SI25. 33. Roberts M, Hsiao W, Berman P, Reich M. Geting health reform right. A guide to improving performance and equity. New York: Oxford University Press, 2004.

34. Programa de Naciones Unidas para el Desarrollo. La democracia en América Latina. Hacia una democracia de ciudadanas y ciudadanos. Buenos Aires: Santillana, 2004. 